\title{
A Novel Neural Network Model for Extracting the Largest Sum of Real Part and Imaginary Part of Eigenvalues and the Corresponding Eigenvectors of a Real Matrix
}

\author{
Hang Tan, Xue-Song Liang, Li-Ping Wan, Zhao-Yao Wu \\ College of Physics and Engineering, Chengdu Normal University (CDNU), Chengdu, China \\ E-mail: hangtancdnu@163.com,liang_xuesong@sohu.com, lpwan2013@126.com
}

\begin{abstract}
In this study, we proposed a novel complex neural network model, which extends the neural networks based approaches that can asymptotically compute the largest imaginary part or the largest real part of eigenvalues to the case of directly calculating the largest sum of real part and imaginary part of eigenvalues and the corresponding eigenvectors of a real matrix. The proposed neural network algorithm is described by a group of complex differential equations. And the algorithm has parallel processing ability in an asynchronous manner and could achieve high computing capability. This paper also provides a rigorous mathematical proof for its convergence for a more clear understanding of network dynamic behaviors relating to the computation of the eigenvector and the eigenvalue. Numerical examples showed that the proposed algorithm has good performance for a general real matrix.
\end{abstract}

Keywords- complex neural network; real matrix; maximum sum of real part and imaginary part; eigenvalue; eigenvector

\section{INTRODUCTION}

Using the neural network technology to extract eigenpairs of matrices is presented about 30 years [1], and then motivated broad interests from engineering and theoretical researches [2-12]. In 1995, Luo et al. [5-7] proposed a very classical neural network algorithm for extracting not only modulus maximum eigenvalue but also modulus minimum eigenvalue and their corresponding eigenvectors of real symmetric matrices. In the very recent years, some adaptive generalized eigen-pairs extraction algorithms of Hermite matrices have been developed by some authors [11, 12].

A number of years ago, Liu et al. [3] proposed a simpler neural network algorithm for extracting the eigenvector corresponding to the modulus maximum eigenvalue of a real symmetric matrix, in the paper [4], they extended this model to extract the imaginary part of the eigenvalue from the maximum imaginary part and the real part of the eigenvalue from the maximum real part of a general real matrix. In the present paper, we also along with this way, however, we intend try to extract the eigenvalue and the corresponding eigenvector from the largest sum of the real part and the imaginary part of eigenvalues of a general real matrix.

The rest of this paper is organized as follows: In section 2, we proposed the novel complex domain neural network model for our purpose. Some numerical examples were given in section 3 and we summarized this paper in the last section.

\section{THE NOVEL COMPLEX NEURAL NETWORK MODEL}

We consider the following model in [3, 4]:

$$
\frac{d v(t)}{d t}=A v(t)-|v(t)|^{2} v(t)
$$

In which $v(t) \in R^{n}$ are the $n$ dimension real column vectors that denote the states of neurons. However, this neural network algorithm can only be used to solve the eigen-pair problems of real symmetric matrices. In [4], authors extended this model to compute the largest real part and the largest imaginary part of eigenvalues from a real matrix. In this paper, we intended to extract the eigenvalue and the corresponding eigenvector from the largest sum of the real part and the imaginary part of eigenvalue of a real matrix.

In (1), we instead of the matrix $A$ by $A^{\prime}$ :

$$
A^{\prime}=\left(\begin{array}{cc}
A & A \\
-A & A
\end{array}\right) \text {. }
$$

Where $A$ is a general real matrix, $v(t) \in R^{2 n}$, and assume that

$$
v(t)=\left[\begin{array}{l}
x(t) \\
y(t)
\end{array}\right],
$$

We can get $v(t)^{T}=\left[x(t)^{T}, y(t)^{T}\right]$, so (1) can be converted into the following forms:

$$
\begin{aligned}
& \frac{d x(t)}{d t}=[A x(t)+A y(t)]-\sum_{j=1}^{n}\left[x_{j}^{2}+y_{j}^{2}\right] x(t), \\
& \frac{d y(t)}{d t}=[-A x(t)+A y(t)]-\sum_{j=1}^{n}\left[x_{j}^{2}+y_{j}^{2}\right] y(t) .
\end{aligned}
$$

$$
\text { Let } z(t)=x(t)+y(t) \mathrm{i} \text {, it is easily from (2) that: }
$$




$$
\frac{d z(t)}{d t}=(1-i) A z(t)-z^{T}(t) \bar{z}(t) z(t)
$$

Equation (3) is the complex neural network model in our study, where $z(t) \in C^{n}$. The algorithm can be used to extract the eigenvector and the corresponding eigenvalue, which has largest real part and imaginary part sum.

All eigenvalues of real matrix $A$ can be denoted as $\lambda_{1}^{R}+\lambda_{1}^{I} i, \ldots, \lambda_{n}^{R}+\lambda_{n}^{I} i, \quad\left(\lambda_{k}^{R}, \lambda_{k}^{R} \in R, k=1,2, \ldots, n\right) \quad$, the corresponding normalized complex eigenvectors are denoted as $S_{1}, S_{2}, \cdots, S_{n}$, obviously, they construct a normalized basis in $C^{n \times n}$. Assume that

$$
z(t)=\sum_{k=1}^{n} z_{k}(t) S_{k}=\sum_{k=1}^{n}\left[x_{k}(t)+\mathrm{i} y_{k}(t)\right] S_{k}
$$

Where $z_{k}(t)=x_{k}(t)+\mathrm{i} y_{k}(t)$ denote the projection of $z(t)$ in the direction along with $S_{k}$.

Theorem 1. Let $z_{k}(t)=x_{k}(t)+\mathrm{i} y_{k}(t)$ denote the projection of $z(t)$ in the direction along with $S_{k}$, the analytic expression of $|z(t)|^{2}$ can be read as

$$
|z(t)|^{2}=\frac{\sum_{k=1}^{n} \exp \left[2\left(\lambda_{k}^{R}+\lambda_{k}^{I}\right) t\right] \times\left|z_{k}(0)\right|^{2}}{1+2 \sum_{j=1}^{n}\left|z_{j}(0)\right|^{2} \int_{0}^{t} \exp \left[2\left(\lambda_{j}^{R}+\lambda_{j}^{I}\right) \tau\right] d \tau} .
$$

Proof: We first need to prove that the projection of $z(t)$ onto $S_{k}$ can be represented as:

$$
\left|z_{k}(t)\right|^{2}=\frac{\exp \left[2\left(\lambda_{k}^{R}+\lambda_{k}^{I}\right) t\right] \times\left|z_{k}(0)\right|^{2}}{1+2 \sum_{j=1}^{n}\left|z_{j}(0)\right|^{2} \int_{0}^{t} \exp \left[2\left(\lambda_{j}^{R}+\lambda_{j}^{I}\right) \tau\right] d \tau},
$$

Assume that all eigenvalues of $A$ can be denoted as $\lambda_{k}^{R}+\mathrm{i} \lambda_{k}^{I}, k=1, \cdots, n$, where $\lambda_{k}^{R}$ and $\lambda_{k}^{I}$ are the real part and the imaginary part of the eigenvalue $\lambda_{k}$, respectively. And also assume that all the corresponding eigenvectors $S_{1}, S_{2}, \cdots, S_{n}$ construct a normalized basis.

Substituting Eq. 4 into Eq. 3, by use of $A S_{k}=\lambda_{k} S_{k}$, one can get

$$
\begin{aligned}
& \sum_{k=1}^{n}\left[\frac{d x_{k}(t)}{d t}+\mathrm{i} \frac{d y_{k}(t)}{d t}\right] S_{k}=(1-i) \sum_{k=1}^{n} \lambda_{k}\left(x_{k}+\mathrm{i} y_{k}\right) S_{k} \\
& -\sum_{j=1}^{n}\left|z_{j}(t)\right|^{2} \sum_{k=1}^{n}\left(x_{k}+\mathrm{i} y_{k}\right) S_{k} .
\end{aligned}
$$

Along with $S_{k}$, the following equation can be obtained:

$$
\begin{aligned}
& \frac{d x_{k}(t)}{d t}+\mathrm{i} \frac{d y_{k}(t)}{d t}=(1-i) \lambda_{k}\left(x_{k}+\mathrm{i} y_{k}\right) \\
& -\sum_{j=1}^{n}\left|Z_{j}(t)\right|^{2}\left(x_{k}+\mathrm{i} y_{k}\right) .
\end{aligned}
$$

Substitute of $\lambda_{j}=\lambda_{j}^{R}+\mathrm{i} \lambda_{j}^{I}, \overline{\lambda_{j}}=\lambda_{j}^{R}-\mathrm{i} \lambda_{j}^{I} \quad$ and $\lambda_{k}=\lambda_{k}^{R}+\mathrm{i} \lambda_{k}^{I}$ into (8). After separating the real part and the imaginary part, we have:

$$
\begin{aligned}
& \frac{d x_{k}(t)}{d t}=\left(x_{k}+y_{k}\right) \lambda_{k}^{R}+\left(x_{k}-y_{k}\right) \lambda_{k}^{I}-\sum_{j=1}^{n}\left|z_{j}\right|^{2} x_{k}, \\
& \frac{d y_{k}(t)}{d t}=\left(x_{k}+y_{k}\right) \lambda_{k}^{I}-\left(x_{k}-y_{k}\right) \lambda_{k}^{R}-\sum_{j=1}^{n}\left|z_{j}\right|^{2} y_{k} .
\end{aligned}
$$

As $\frac{d \mid z_{k}{ }^{2}}{d t}=\frac{d\left(\overline{z_{k}} z_{k}\right)}{d t}=2 x_{k} \frac{d x_{k}}{d t}+2 y_{k} \frac{d y_{k}}{d t}$, based on (9), we can obtain the following equation:

$$
\frac{d\left|z_{k}(t)\right|^{2}}{d t}=2\left|z_{k}\right|^{2}\left(\lambda_{k}^{R}+\lambda_{k}^{I}\right)-2 \sum_{j=1}^{n}\left|z_{j}\right|^{2}\left|z_{k}\right|^{2} .
$$

From Eq. (10), if $\left|z_{k}(t)\right|^{2} \neq 0,\left|z_{r}(t)\right|^{2} \neq 0$, then

$$
\begin{aligned}
& \frac{1}{\left|z_{k}(t)\right|^{2}} \frac{d}{d t}\left|z_{k}(t)\right|^{2}-\frac{1}{\left|z_{r}(t)\right|^{2}} \frac{d}{d t}\left|z_{r}(t)\right|^{2} \\
& =2\left[\left(\lambda_{k}^{R}+\lambda_{k}^{I}\right)-\left(\lambda_{r}^{R}+\lambda_{r}^{I}\right)\right]
\end{aligned}
$$

Therefore

$$
\frac{d}{d t}\left\{\ln \frac{\left|z_{k}(t)\right|^{2}}{\left|z_{r}\right|^{2}}\right\}=2\left[\left(\lambda_{k}^{R}+\lambda_{k}^{I}\right)-\left(\lambda_{r}^{R}+\lambda_{r}^{I}\right)\right]
$$

i.e.

$$
\frac{\left|z_{k}\right|^{2}}{\left|z_{r}\right|^{2}}=\frac{\left|z_{k}(0)\right|}{\left|z_{r}(0)\right|^{2}} \exp \left\{2\left[\left(\lambda_{k}^{R}+\lambda_{k}^{I}\right)-\left(\lambda_{r}^{R}+\lambda_{r}^{I}\right)\right] t\right\} .
$$

From (10), we can directly write it as follows:

$$
\frac{1}{\left|z_{k}\right|^{4}} \frac{d}{d t}\left|z_{k}\right|^{2}=2\left(\lambda_{k}^{R}+\lambda_{k}^{I}\right) \frac{1}{\left|z_{k}\right|^{2}}-2 \sum_{j=1}^{n} \frac{\left|z_{j}\right|^{2}}{\left|z_{k}\right|^{2}},
$$

i.e.

$$
\frac{d}{d t} \frac{1}{\left|z_{k}(t)\right|^{2}}+2\left(\lambda_{k}^{R}+\lambda_{k}^{I}\right) \frac{1}{\left|z_{k}(t)\right|^{2}}=2 \sum_{j=1}^{n} \frac{\left|z_{j}(t)\right|^{2}}{\left|z_{k}(t)\right|^{2}}
$$

By use of (12), one gets the following (15) from the above (14): 


$$
\frac{d}{d t} \frac{\exp \left[2\left(\lambda_{k}^{R}+\lambda_{k}^{I}\right) t\right]}{\left|z_{k}(t)\right|^{2}}=2 \sum_{j=1}^{n} \frac{\left|z_{j}(0)\right|^{2}}{\left|z_{k}(0)\right|^{2}} \exp \left[2\left(\lambda_{j}^{R}+\lambda_{j}^{I}\right) t\right]
$$

The integral on both sides of (15) from 0 to $t$ can reads

$$
\begin{aligned}
& \frac{\exp \left[2\left(\lambda_{k}^{R}+\lambda_{k}^{I}\right) t\right]}{\left|z_{k}(t)\right|^{2}}-\frac{1}{\left|z_{k}(0)\right|^{2}}= \\
& \quad 2 \sum_{j=1}^{n} \frac{\left|z_{j}(0)\right|^{2}}{\left|z_{k}(0)\right|^{2}} \int_{0}^{t} \exp \left[2\left(\lambda_{j}^{R}+\lambda_{j}^{I}\right) \tau\right] d \tau .
\end{aligned}
$$

Direct calculation reads

$$
\left|z_{k}(t)\right|^{2}=\frac{\exp \left[2\left(\lambda_{k}^{R}+\lambda_{k}^{I}\right) t\right] \times\left|z_{k}(0)\right|^{2}}{1+2 \sum_{j=1}^{n}\left|z_{j}(0)\right|^{2} \int_{0}^{t} \exp \left[2\left(\lambda_{j}^{R}+\lambda_{j}^{I}\right) \tau\right] d \tau},
$$

therefore one gets (5).

If an equilibrium vector of RNN (3) exists, let $\xi$ denote it, and there exists

$$
\xi=\lim _{t \rightarrow \infty} z(t)
$$

Theorem 2: If A is not a zero matrix, then $|\xi| \neq 0$.

Proof: Obviously, from the theorem 1 , we have

$$
|\xi|=\lim _{t \rightarrow \infty}|z(t)|=\lim _{t \rightarrow \infty} \sqrt{\frac{\sum_{k=1}^{n} \exp \left[2\left(\lambda_{k}^{R}+\lambda_{k}^{I}\right) t\right] \times\left|z_{k}(0)\right|^{2}}{1+2 \sum_{j=1}^{n}\left|z_{j}(0)\right|^{2} \int_{0}^{t} \exp \left[2\left(\lambda_{j}^{R}+\lambda_{j}^{I}\right) \tau\right] d \tau}},
$$

$|\xi|=0$ implies that all eigenvalues of A are 0 , so A should be a zero matrix.

Theorem 3: If each eigenvalue of $\mathrm{A}$ satisfies that $\lambda_{k}^{R}+\lambda_{k}^{I}<0(k=1,2, \ldots, n)$, then $|\xi|=0$.

Proof: From the theorem 1 and the theorem 2, we have that $\sum_{k=1}^{n} \exp \left[2\left(\lambda_{k}^{R}+\lambda_{k}^{I}\right) t\right] \times\left|z_{k}(0)\right|^{2}=0$, so $|\xi|=0$. In this case, the model (3) can't work correctly in this case.

Theorem 4: Denote $\left(\lambda_{m}^{R}+\lambda_{m}^{I}\right)=\max _{1 \leq k \leq n}\left(\lambda_{k}^{R}+\lambda_{k}^{I}\right)$. If $\left(\lambda_{m}^{R}+\lambda_{m}^{I}\right) \geq 0$, then $\xi^{T} \bar{\xi}=\left(\lambda_{m}^{R}+\lambda_{m}^{I}\right)$.

Proof: From (17) and the theorem 1, we have

$$
\begin{aligned}
& \xi^{T} \bar{\xi}=\lim _{t \rightarrow \infty}|z(t)|^{2}=\lim _{t \rightarrow \infty} \frac{\sum_{k=1}^{n} \exp \left[2\left(\lambda_{k}^{R}+\lambda_{k}^{I}\right) t\right] \times\left|z_{k}(0)\right|^{2}}{1+2 \sum_{j=1}^{n}\left|z_{j}(0)\right|^{2} \int_{0}^{t} \exp \left[2\left(\lambda_{j}^{R}+\lambda_{j}^{I}\right) \tau\right] d \tau} \\
& =\lim _{t \rightarrow \infty} \frac{E_{1}}{E_{2}+E_{3}} \\
& =\left(\lambda_{m}^{R}+\lambda_{m}^{I}\right),
\end{aligned}
$$

Where

$$
\begin{aligned}
& E_{1}=\left|z_{m}(0)\right|^{2}+\sum_{k=1, k \neq m}^{n} \exp \left[2\left(\lambda_{k}^{R}+\lambda_{k}^{I}-\lambda_{m}^{R}-\lambda_{m}^{I}\right) t\right] \times\left|z_{k}(0)\right|^{2}, \\
& E_{2}=\exp \left[-2\left(\lambda_{m}^{R}+\lambda_{m}^{I}\right) t\right]\left\{1+2 \sum_{j=1, j \neq m}^{n}\left|z_{j}(0)\right|^{2} \int_{0}^{t} \exp \left[2\left(\lambda_{j}^{R}+\lambda_{j}^{I}\right) \tau\right] d \tau\right\}, \\
& E_{3}=\frac{1}{\left(\lambda_{m}^{R}+\lambda_{m}^{I}\right)}\left|z_{m}(0)\right|^{2}\left\{1-\exp \left[-2\left(\lambda_{m}^{R}+\lambda_{m}^{I}\right) t\right]\right\} .
\end{aligned}
$$

The theorem is proved. From this theorem, we know that when the largest sum of real part and imaginary part of eigenvalues is positive, model (3) will converge to a nonzero equilibrium vector, in addition, the square modulus of the vector is equal to the largest sum of real part and imaginary part of all eigenvalues.

Theorem 5: For RNN model (3), if there are no exist two or more different eigenvalues shared the same largest sum of real part and the imaginary part of eigenvalues, then $\lim _{t \rightarrow \infty} \frac{\bar{z}^{T}(t) A z(t)}{\bar{Z}^{T}(t) z(t)}$ will converge to the eigenvalue that has the largest sum of the real part and the imaginary part, and the corresponding eigenvector is $\xi=\lim _{t \rightarrow \infty} z(t)$.

Proof: From the theorem 1, we have

$$
\begin{aligned}
& \lim _{t \rightarrow \infty} \frac{\bar{Z}^{T}(t) A z(t)}{\bar{Z}^{T}(t) z(t)}=\frac{\sum_{l=1}^{n} \bar{S}_{l}^{T} \bar{z}_{l}^{T} A \sum_{k=1}^{n} z_{k} S_{k}}{\sum_{l=1}^{n} \bar{S}_{l}^{T} \bar{Z}_{l}^{T} \sum_{k=1}^{n} z_{k} S_{k}} \\
& =\frac{\sum_{l=1}^{n} \bar{S}_{l}^{T} \bar{z}_{l}^{T} \sum_{k=1}^{n} z_{k} \lambda_{k} S_{k}}{\sum_{l=1}^{n} \bar{S}_{l}^{T} \bar{z}_{l}^{T} \sum_{k=1}^{n} z_{k} S_{k}}=\frac{\sum_{l=1}^{n} \sum_{k=1}^{n} \lambda_{k} \bar{z}_{l}^{T} z_{k} \bar{S}_{l}^{T} S_{k}}{\sum_{l=1}^{n} \sum_{k=1}^{n} \bar{z}_{l}^{T} z_{k} \bar{S}_{l}^{T} S_{k}} \\
& =\frac{\lambda_{m}+\sum_{j=1, j \neq m}^{n} \lambda_{j} \frac{\bar{Z}_{j}^{T} Z_{j}}{\bar{Z}_{m}^{T} Z_{m}}+\sum_{l=1}^{n} \sum_{k=1, k \neq l}^{n} \lambda_{k} \frac{\bar{Z}_{l}^{T} Z_{k}}{\bar{Z}_{m}^{T} Z_{m}} \bar{S}_{l}^{T} S_{k}}{\bar{Z}_{j}^{T} Z_{j}} \\
& 1+\sum_{j=1, j \neq m}^{n} \frac{\bar{Z}_{j}^{T} Z_{j}}{\bar{Z}_{m}^{T} Z_{m}}+\sum_{l=1}^{n} \sum_{k=1, k \neq l}^{n} \frac{\bar{z}_{l}^{T} Z_{k}}{\bar{z}_{m}^{T} Z_{m}} \bar{S}_{l}^{T} S_{k} \\
& =\frac{\lambda_{m}+\sum_{j=1, j \neq m}^{n} \lambda_{j} \frac{\left|z_{j}\right|^{2}}{\left|z_{m}\right|^{2}}+\sum_{l=1}^{n} \sum_{k=1, k \neq l}^{n} \lambda_{k} \frac{\bar{z}_{l}^{T} z_{k}}{\bar{z}_{m}^{T} Z_{m}} \bar{S}_{l}^{T} S_{k}}{1+\sum_{j=1, j \neq m}^{n} \frac{\left|z_{j}\right|^{2}}{\left|Z_{m}\right|^{2}}+\sum_{l=1}^{n} \sum_{k=1, k \neq l}^{n} \frac{\bar{z}_{l}^{T} z_{k}}{\bar{Z}_{m}^{T} Z_{m}} \bar{S}_{l}^{T} S_{k}}
\end{aligned}
$$

Based on (12) we have

$$
\begin{aligned}
& \lim _{t \rightarrow \infty} \frac{\left|z_{j}(t)\right|^{2}}{\left|z_{m}(t)\right|^{2}}=\lim _{t \rightarrow \infty} \frac{\left|z_{j}(0)\right|^{2}}{\left|z_{m}(0)\right|^{2}} \exp \left\{2\left[\left(\lambda_{j}^{R}+\lambda_{j}^{I}\right)-\left(\lambda_{m}^{R}+\lambda_{m}^{I}\right)\right] t\right\}=0, \\
& \text { so } \sum_{j=1, j \neq m}^{n} \lambda_{j} \frac{\left|z_{j}\right|^{2}}{\left|z_{m}\right|^{2}}=0 \text {. In addition, from Eq. (6), we }
\end{aligned}
$$

have $\lim _{t \rightarrow \infty} z_{k}(t)=0, k \neq m$, one gets 


$$
\begin{aligned}
& 0 \leq \lim _{t \rightarrow \infty} \frac{\bar{z}_{l}^{T}(t) z_{k}(t)}{\bar{z}_{m}^{T}(t) z_{m}(t)} \leq \lim _{t \rightarrow \infty} \frac{\frac{1}{2}\left[\bar{z}_{l}^{T}(t) z_{l}(t)+\bar{z}_{k}^{T}(t) z_{k}(t)\right]}{\bar{z}_{m}^{T}(t) z_{m}(t)} \\
& =\lim _{t \rightarrow \infty} \frac{1}{2}\left(\frac{\left|z_{l}(t)\right|^{2}}{\left|z_{m}(t)\right|^{2}}+\frac{\left|z_{k}(t)\right|^{2}}{\left|z_{m}(t)\right|^{2}}\right) \\
& =\lim _{t \rightarrow \infty} \frac{1}{2}\left(\frac{\left|z_{l}(0)\right|^{2}}{\left|z_{m}(0)\right|^{2}} \exp \left\{2\left[\left(\lambda_{l}^{R}+\lambda_{l}^{I}\right)-\left(\lambda_{m}^{R}+\lambda_{m}^{I}\right)\right] t\right\}\right. \\
& \left.+\frac{\left|z_{k}(0)\right|^{2}}{\left|z_{m}(0)\right|^{2}} \exp \left\{2\left[\left(\lambda_{k}^{R}+\lambda_{k}^{I}\right)-\left(\lambda_{m}^{R}+\lambda_{m}^{I}\right)\right] t\right\}\right) \\
& =0 . \\
& \sum_{l=1}^{n} \sum_{k=1, k \neq l}^{n} \lambda_{k} \frac{\bar{z}_{l}^{T} z_{k}}{\bar{z}_{m}^{T} z_{m}} \bar{S}_{l}^{T} S_{k}=0, \sum_{l=1}^{n} \sum_{k=1, k \neq l}^{n} \frac{\bar{l}_{l}^{T} z_{k}}{\bar{z}_{m}^{T} z_{m}} \bar{z}_{l}^{T} z_{k} \bar{S}_{l}^{T} S_{k}=0 .
\end{aligned}
$$

We have

$$
\lim _{t \rightarrow \infty} \frac{\bar{Z}^{T}(t) A z(t)}{\bar{Z}^{T}(t) Z(t)}=\lambda_{m},
$$

Further, one gets

$$
\begin{aligned}
& \lim _{t \rightarrow \infty} \bar{Z}^{T}(t) A z(t)=\lambda_{m} \lim _{t \rightarrow \infty} \bar{Z}^{T}(t) z(t), \\
& \lim _{t \rightarrow \infty} \bar{Z}^{T}(t)\left[A z(t)-\lambda_{m} z(t)\right]=0,
\end{aligned}
$$

from the theorem 2, $\lim _{t \rightarrow \infty} \bar{Z}^{T}(t) \neq 0$, we have $\lim _{t \rightarrow \infty}\left[A z(t)-\lambda_{m} z(t)\right]=0$, directly we can get $A \lim _{t \rightarrow \infty} z(t)=\lambda_{m} \lim _{t \rightarrow \infty} z(t)$, so $\xi=\lim _{t \rightarrow \infty} z(t)$ is the eigenvector corresponding to the eigenvalue $\lambda_{m}$, which has the largest sum of the real part and the imaginary part.

\section{Simulation Results}

Consider the following $6 \times 6$ real artificial matrix $A$ :

$$
A=\left(\begin{array}{rrrrrr}
-0.3403 & 1.1186 & -0.4837 & 0.1067 & 3.2967 & -0.5119 \\
1.3411 & -0.0051 & 1.1793 & -0.1264 & -0.4837 & 0.3074 \\
0.1321 & -0.4286 & -1.0927 & 0.8038 & 0.3281 & -0.7417 \\
0.2983 & 0.0407 & 0.0974 & -0.9738 & 0.5583 & 0.7618 \\
0.9101 & 0.5137 & -1.3900 & -1.6347 & 0.7752 & 1.2038 \\
-1.1125 & 0.0847 & 0.2311 & 1.3535 & -0.7823 & 1.5441
\end{array}\right)
$$

The six eigenvalues of $A$ are :

$$
1.8901 \pm 0.8337 \mathrm{i},-2.4700,0.0134 \pm 1.1816 \mathrm{i},-1.4297
$$

We use the following stochastic initial value $z(0)$ for running the complex neural network algorithm (3) to obtain the largest sum of real part and imaginary part eigenvalues of matrix $A$ :

$$
z(0)=(0.5,0.5,0.5,0.5,0.5,0.5)^{T}
$$

We can obtain the eigenvectors corresponding to the maximum sum of real part and imaginary part of eigenvalues of $A$, as follows:

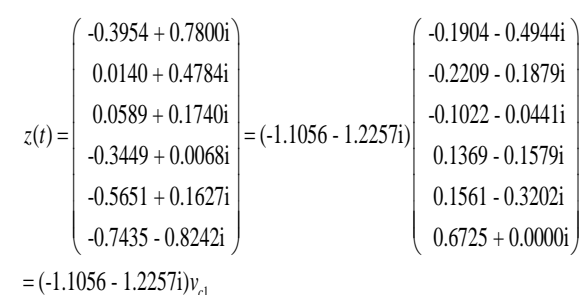

From the above, the eigenvector that we get is constant multiple of the eigenvector obtained from the direct calculation $v_{c 1}$. The corresponding eigenvalue is $\lambda=\frac{\overline{z(t)}^{T} A z(t)}{\overline{z(t)}^{T} z(t)}=1.8901+0.8337 \mathrm{i}$, which is just the largest sum of real part and imaginary part eigenvalues of $A$.

Fig. 1 illustrates the dynamic behavior of the modulus of largest sum of real part and imaginary part eigenvalues of A, and Fig. 2 illustrates the dynamic behavior of six components' modulus of the corresponding eigenvector. From these figures, we note that the proposed algorithm also has the fast convergence property, which is just one virtue of parallel computing. In addition, the proposed algorithm is not sensitive to initial value. This virtue makes actual operation a lot more convenient.

\section{CONCLUSION}

Based on the classical real domain neural network, this paper proposed a novel complex neural network to directly compute the largest sum of real part and imaginary part of eigenvalues and the corresponding eigenvectors of real matrices. Simulation experiment indicated that the proposed algorithm is effective.

\section{ACKNOWLEDGMENT}

This work was partially supported by the General project of Sichuan Provincial Department of Education (14ZB0331), and The Chengdu Normal University introduces the talented person scientific research start funds subsidization project (YJRC2014-5).

\section{REFERENCES}

[1] Oja E, "A simplified neuron algorithm as a principal component analyzer,” Journal of Mathematical Biology ,vol.15,1982,pp.267-273.

[2] Chen Tianping, "Principal component analysis, neural networks and eigenvalues of matrices,” Chinese Sci Bull ,vol.41,1995,pp.13441344. 
[3] Y. Liu, Z.S. You and L.P. Cao, “ A concise functional neural network computing the largest modulus eigenvalues and their corresponding eigenvectors of a real skew matrix," Theoretical Computer Science ,vol.367,2006,pp. 273-285.

[4] Yiguang Liu, Zhisheng You and Liping Cao, “A Recurrent Neural Network Computing the Largest Imaginary or Real Part of Eigenvalues of Real Matrices,” Computers and Mathematics with Applications, vol.53,2007,pp.41-53.

[5] F.L. Luo, Y.D. Li, "Real-time neural computation of the eigenvector corresponding to the largest eigenvalue of positive matrix," Neurocomputing ,vol.7,1995,pp.145-157.

[6] F.L. Luo, R. Unbehauen and Y.D. Li, "A principal component analysis algorithm with invariant norm," Neurocomputing ,vol.8,1995,pp.213-221.

[7] Y. Zhang, F. Yan, and H.J. Tang, "Neural networks based approach for computing eigenvectors and eigenvalues of symmetric matrix," Comput. Math. Appl, vol.47, 2004, pp.1155-1164.

[8] Y. Liu, Z.S. You and L.P. Cao, "A functional neural network for computing the largest modulus eigenvalues and their corresponding eigenvectors of an anti-symmetric matrix,” Neurocomputing, vol.67, 2005,pp.384-397.

[9] Y. Liu, Z.S. You and L.P. Cao, "A concise functional neural network computing the largest modulus eigenvalues and their corresponding eigenvectors of a real skew matrix,” Theoretical Computer Science , vol. 367, 2006, pp.273-285.

[10] Ying Tang and J.P. Li, "Another neural network based approach for computing eigenvalues and eigenvectors of real skew-symmetric matrices," Computers and Mathematics with Applications,vol. 60, 2010, pp.1385-1392.

[11] T. D. Nguye and I. Yamada, “Adaptive normalized quasi-Newton algorithms for extraction of generalized eigen-pairs and their convergence analysis,” IEEE Trans. Signal Process, vol. 61, 2013, pp.1404-1418.

[12] Xiaowei Feng, Xiangyu Kong, Zhansheng Duan and Hongguang Ma, "Adaptive Generalized Eigen-Pairs Extraction Algorithms and Their Convergence Analysis,” IEEE TRANSACTIONS ON SIGNAL PROCESSING, vol. 64, 2016, pp.2976-2989

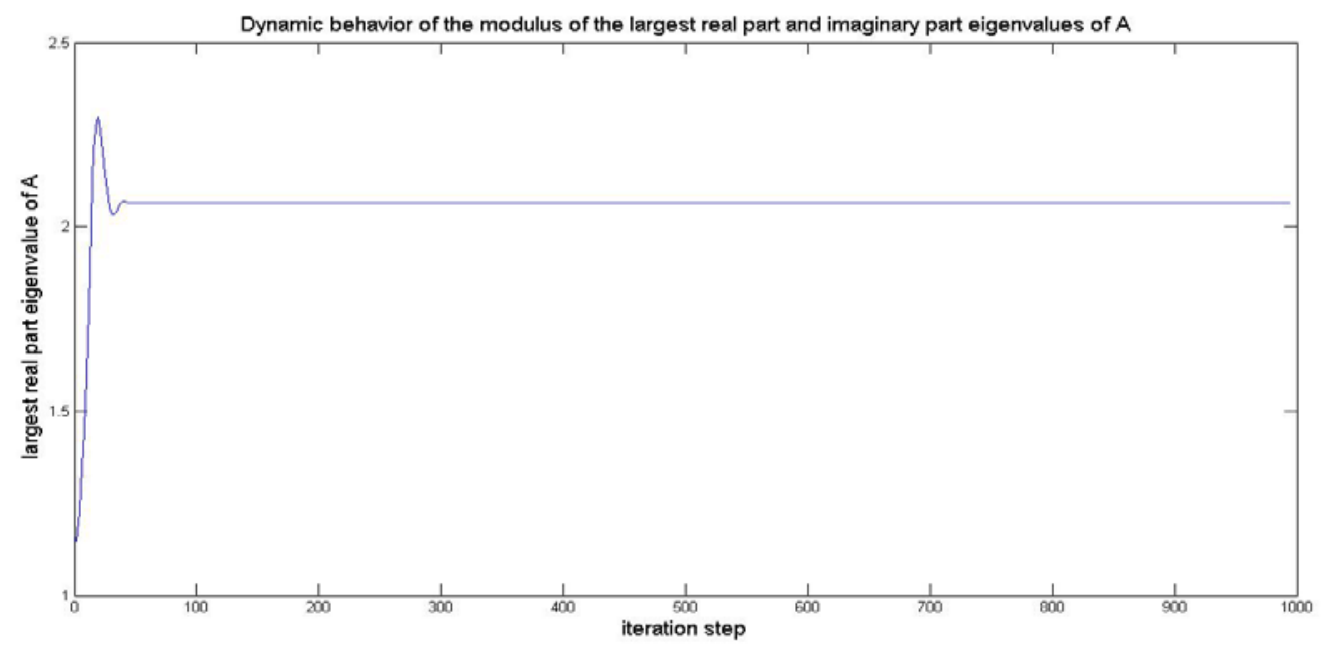

Figure 1. Dynamic behavior of the modulus of the maximum real part and imaginary part eigenvalues of $A$. It should converge to $\left|\lambda_{1}\right|=|1.8901+0.8337 i|=2.0658$.

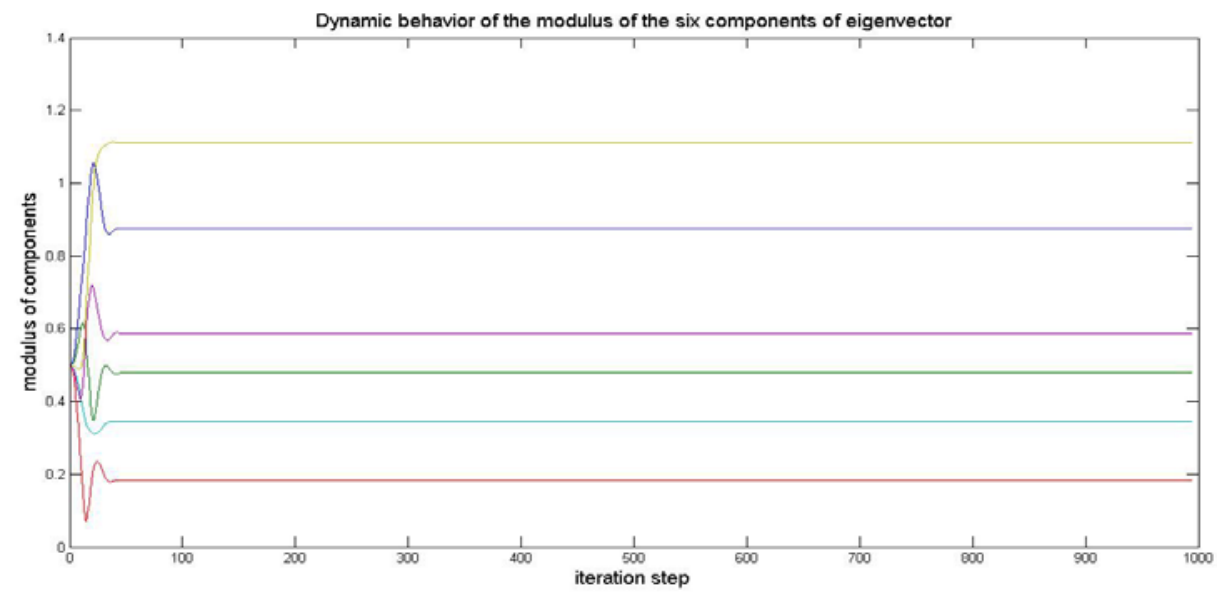

Figure 2. Dynamic behavior of the modulus of the six components of eigenvector corresponding to the maximum real part eigenvalue. It should converge to the six components' modulus of eigenvector corresponding to eigenvalue $\lambda_{1}=1.8901+0.8337 \mathrm{i}$. 